\begin{tabular}{lll}
\hline upf. & $\begin{array}{l}\text { Universitat } \\
\text { Pompeu Fabra } \\
\text { Barcelona }\end{array}$ & $\begin{array}{l}\text { Department } \\
\text { of Economics and Business }\end{array}$
\end{tabular}

Economics Working Paper Series

Working Paper No. 1595

\title{
Inequality reducing properties of income tax schedules
}

Oriol Carbonell-Nicolau and Humberto Llavador

January 2018 


\title{
Inequality Reducing Properties of Income Tax Schedules
}

\author{
Oriol Carbonell-Nicolau* Humberto Llavador ${ }^{\dagger}$
}

January 2018

\begin{abstract}
In a classic result, Jakobsson (1976) and Fellman (1976) showed that average-rate progressive, and only average-rate progressive, income taxes reduce income inequality. Carbonell-Nicolau and Llavador (forthcoming) extended this result to the case of endogenous income, showing that marginal-rate progressivity-in the sense of increasing marginal tax rates on income-is necessary for tax structures to be inequality reducing, and obtaining necessary and sufficient conditions on the social utility function under which progressive and only progressive taxes are inequality reducing. Building on Carbonell-Nicolau and Llavador (forthcoming), this paper furnishes conditions on primitives under which various subclasses of progressive taxes are inequality reducing. The main results in Carbonell-Nicolau and Llavador (forthcoming) are obtained as particular cases of the more general framework presented here. Restricting the set of taxes allows for larger classes of preferences consistent with inequality reducing income taxation. As an illustration of the results' practical implications, we provide a precise characterization of the subclass of (progressive) taxes that are inequality reducing for some standard families of preferences.
\end{abstract}

Keywords: progressive taxation; income inequality; incentive effects of taxation. JEL classifications: D63, D71.

\section{Introduction}

The link between income inequality and progressive taxation uncovered in the seminal works of Jakobsson (1976) and Fellman (1976) has long been considered a fundamental normative foundation for income tax progressivity. ${ }^{1}$ In a recent paper, Carbonell-Nicolau and

\footnotetext{
*Department of Economics, Rutgers University, 75 Hamilton St., New Brunswick, NJ 08901. E-mail: carbonell-nicolau@rutgers . edu.

${ }^{\dagger}$ Universitat Pompeu Fabra and Barcelona GSE, R. Trias Fargas 25-27, 08005 Barcelona, Spain. E-mail: humberto. llavador@upf .edu

${ }^{1}$ The literature on the redistributive effects of tax systems was initiated by Musgrave and Thin (1948). The contributions of Jakobsson (1976) and Fellman (1976) led to a large body of literature on the foundations of tax
} 
Llavador (forthcoming) extended the classic result of Jakobsson (1976) and Fellman (1976)_ according to which average-rate progressive, and only average-rate progressive income taxes, reduce income inequality - to the case of endogenous income. There it was shown that marginal-rate progressivity - in the sense of increasing marginal tax rates on income-is a necessary condition for tax structures to be inequality reducing, and necessary and sufficient conditions on the social utility function were identified under which progressive and only progressive taxes are inequality reducing. While this result circumvents the difficulties and the negative results emphasized by other authors in their attempts to incorporate the disincentive effects of taxation (see, e.g., Allingham (1979) and Ebert and Moyes (2003, 2007)), it remains silent about the families of social utility functions for which subclasses of the set of all progressive tax schedules are inequality reducing. This paper identifies necessary and sufficient conditions on social preferences ensuring that various subclasses of progressive taxes are inequality reducing. The results obtained here are strict generalizations of those in Carbonell-Nicolau and Llavador (forthcoming) and confer a degree of useful flexibility on the theory, in that they allow the analyst to expand the universe of social preferences by suitably restricting the set of progressive taxes. As an illustration of the results' practical implications, we provide a precise characterization of the subclass of (progressive) taxes that are inequality reducing for some standard families of preferences.

\section{Preliminaries}

The setting is the same as that of Carbonell-Nicolau and Llavador (forthcoming). There are $n$ individuals. The social utility function is given by a continuous utility function $u: \mathbb{R}_{+} \times[0,1] \rightarrow \mathbb{R}$ defined over consumption-labor pairs $(c, l) \in \mathbb{R}_{+} \times[0,1]$ such that $u(\cdot, l)$ is strictly increasing in $c$ for each $l \in[0,1)$, and $u(c, \cdot)$ is strictly decreasing in $l$ for each $c>0$. The map $u$ is assumed strictly quasiconcave on $\mathbb{R}_{++} \times[0,1)$ and twice continuously differentiable on $\mathbb{R}_{++} \times(0,1)$. For $(c, l) \in \mathbb{R}_{++} \times(0,1)$, let

$$
\operatorname{MRS}(c, l):=-\frac{u_{l}(c, l)}{u_{c}(c, l)}
$$

denote the marginal rate of substitution of labor for consumption, where

$$
u_{c}(c, l):=\frac{\partial u(c, l)}{\partial c} \quad \text { and } \quad u_{l}(c, l):=\frac{\partial u(c, l)}{\partial l}
$$

We assume that for each $c>0$,

$$
\lim _{l \rightarrow 1^{-}} M R S(c, l)=+\infty \text { and } \lim _{l \rightarrow 0^{+}} \operatorname{MRS}(c, l)<+\infty .
$$

The set of all utility functions satisfying the above conditions is denoted by $\mathscr{U}$. 
We restrict attention to nondecreasing and order-preserving piecewise linear tax schedules.

Definition 1. Let $\left(\alpha_{0}, \boldsymbol{t}, \overline{\boldsymbol{y}}\right)=\left(\alpha_{0},\left(t_{0}, \ldots, t_{K}\right),\left(\bar{y}_{0}, \ldots, \bar{y}_{K}\right)\right)$, where $\alpha_{0} \geq 0, K \in \mathbb{Z}_{+}, t_{k} \in[0,1)$ for each $k \in\{0, \ldots, K\}, t_{k} \neq t_{k+1}$ whenever $k \in\{0, \ldots, K-1\}$ and $K \geq 1$, and $0=\bar{y}_{0}<\cdots<\bar{y}_{K}$. A $(K+1)$-bracket piecewise linear tax schedule is a real-valued map $T$ on $\mathbb{R}_{+}$uniquely determined by $\left(\alpha_{0}, \boldsymbol{t}, \overline{\boldsymbol{y}}\right)$ as follows:

$$
T(y):=\left\{\begin{array}{lc}
-\alpha_{0}+t_{0} y & \text { if } 0=\bar{y}_{0} \leq y \leq \bar{y}_{1}, \\
-\alpha_{0}+t_{0} \bar{y}_{1}+t_{1}\left(y-\bar{y}_{1}\right) & \text { if } \bar{y}_{1}<y \leq \bar{y}_{2}, \\
\vdots & \vdots \\
-\alpha_{0}+t_{0} \bar{y}_{1}+t_{1}\left(\bar{y}_{2}-\bar{y}_{1}\right)+\cdots+t_{K-1}\left(\bar{y}_{K}-\bar{y}_{K-1}\right)+t_{K}\left(y-\bar{y}_{K}\right) & \text { if } \bar{y}_{K}<y .
\end{array}\right.
$$

Here $T(y)$ is interpreted as the tax liability for gross income level $y$. We write $\left(\alpha_{0}, \boldsymbol{t}, \overline{\boldsymbol{y}}\right)$ and the associated map $T$ interchangeably. Note that for $K=0,\left(\alpha_{0}, t_{0}, \bar{y}_{0}=0\right)$ is a linear tax with intercept $\alpha_{0}$ and marginal tax rate $t_{0}$; for $K=1,\left(\alpha_{0},\left(t_{0}, t_{1}\right),\left(\bar{y}_{0}, \bar{y}_{1}\right)\right)$ is a two-bracket tax with intercept $\alpha_{0}$, marginal tax rates $t_{0}$ and $t_{1}$, and bracket threshold $\bar{y}_{1}$; and so on.

The set of piecewise linear tax schedules is denoted by $\mathscr{T}$.

An ability distribution is a vector $\boldsymbol{a}=\left(a_{1}, \ldots, a_{n}\right) \in \mathbb{R}_{++}^{n}$ such that $a_{1} \leq \cdots \leq a_{n}$. The set of all ability distributions is denoted by $\mathscr{A}$.

An agent of ability $a>0$ who chooses $l \in[0,1]$ units of labor and faces a tax schedule $T \in \mathscr{T}$ consumes $c=a l-T(a l)$ units of the good and obtains a utility of $u(c, l)$. Thus, the agent's problem is

$$
\max _{l \in[0,1]} u(a l-T(a l), l) .
$$

Because the members of $\mathscr{U}$ and $\mathscr{T}$ are continuous, for given $u \in \mathscr{U}, a>0$, and $T \in \mathscr{T}$, the optimization problem in (2) has a solution, although it need not be unique. A solution function is a map $l^{u}: \mathbb{R}_{++} \times \mathscr{T} \rightarrow[0,1]$ such that $l^{u}(a, T)$ is a solution to (2) for each $(a, T) \in \mathbb{R}_{++} \times \mathscr{T}$. The pre-tax and post-tax income functions associated to a solution function $l^{u}$, denoted by $y^{u}: \mathbb{R}_{++} \times \mathscr{T} \rightarrow \mathbb{R}_{+}$and $x^{u}: \mathbb{R}_{++} \times \mathscr{T} \rightarrow \mathbb{R}_{+}$respectively, are given by

$$
y^{u}(a, T):=a l^{u}(a, T) \quad \text { and } \quad x^{u}(a, T):=a l^{u}(a, T)-T\left(a l^{u}(a, T)\right) .
$$

Given $a>0$, let $U^{a}: \mathbb{R}_{+} \times[0, a] \rightarrow \mathbb{R}$ be defined by $U^{a}(c, y):=u(c, y / a)$. For $(c, y, a) \in \mathbb{R}_{++}^{3}$ with $y<a$, define

$$
U_{c}^{a}(c, y):=\frac{\partial U^{a}(c, y)}{\partial c}, \quad U_{y}^{a}(c, y):=\frac{\partial U^{a}(c, y)}{\partial y}, \quad \text { and } \eta^{a}(c, y):=-\frac{U_{y}^{a}(c, y)}{U_{c}^{a}(c, y)} .
$$

The following condition was introduced by Mirrlees (1971, Assumption B, p. 182) and termed agent monotonicity by Seade (1982). 
Definition 2. A utility function $u \in \mathscr{U}$ satisfies agent monotonicity if $\eta^{a}(c, y) \geq \eta^{a^{\prime}}(c, y)$ for each $(c, y) \in \mathbb{R}_{+}^{2}$ and $0<a<a^{\prime}$ with $y<a$.

The set of all the members of $\mathscr{U}$ satisfying agent monotonicity is denoted by $\mathscr{U}^{*}$.

An income distribution is a vector $\boldsymbol{z}=\left(z_{1}, \ldots, z_{n}\right) \in \mathbb{R}_{+}^{n}$ of incomes arranged in increasing order, i.e., $z_{1} \leq \cdots \leq z_{n}$.

Given two income distributions $\boldsymbol{z}=\left(z_{1}, \ldots, z_{n}\right)$ and $\boldsymbol{z}^{\prime}=\left(z_{1}^{\prime}, \ldots, z_{n}^{\prime}\right)$ with $z_{n}, z_{n}^{\prime}>0$, we say that $\boldsymbol{z}$ is at least as equal as $\boldsymbol{z}^{\prime}$ if $\boldsymbol{z}$ Lorenz dominates $\boldsymbol{z}^{\prime}$, i.e., if

$$
\frac{\sum_{i=1}^{k} z_{i}}{\sum_{i=1}^{n} z_{i}} \geq \frac{\sum_{i=1}^{k} z_{i}^{\prime}}{\sum_{i=1}^{n} z_{i}^{\prime}}, \quad \text { for all } k \in\{1, \ldots, n\}
$$

For $u \in \mathscr{U}^{*}$, and given pre-tax and post-tax income functions $y^{u}$ and $x^{u}$, an ability distribution $\boldsymbol{a}=\left(a_{1}, \ldots, a_{n}\right) \in \mathscr{A}$ and a tax schedule $T \in \mathscr{T}$ determine a pre-tax income distribution

$$
\boldsymbol{y}^{u}(\boldsymbol{a}, T):=\left(y^{u}\left(a_{1}, T\right), \ldots, y^{u}\left(a_{n}, T\right)\right)
$$

and a post-tax income distribution

$$
\boldsymbol{x}^{u}(\boldsymbol{a}, T):=\left(x^{u}\left(a_{1}, T\right), \ldots, x^{u}\left(a_{n}, T\right)\right) .^{2}
$$

In the absence of taxation, i.e., if $T \equiv 0$, one has $\boldsymbol{y}^{u}(\boldsymbol{a}, 0)=\boldsymbol{x}^{u}(\boldsymbol{a}, T)$.

The following is the central notion of inequality reducing tax schedule.

Definition 3. Let $u \in \mathscr{U}$. A tax schedule $T \in \mathscr{T}$ is income inequality reducing with respect to $u$, which we denote as $u$-iir, if $\boldsymbol{x}^{u}(\boldsymbol{a}, T)$ Lorenz dominates $\boldsymbol{y}^{u}(\boldsymbol{a}, 0)$ for each ability distribution $\boldsymbol{a}:=\left(a_{1}, \ldots, a_{n}\right) \in \mathscr{A}$ and for each pre-tax and post-tax income functions $y^{u}$ and $x^{u}$.

\section{The results}

The notion of progressivity used here is as follows.

Definition 4. A tax schedule $T \in \mathscr{T}$ is marginal-rate progressive if it is a convex function.

The set of all marginal-rate progressive tax schedules in $\mathscr{T}$ is denoted by $\mathscr{T}_{\text {prog }}$.

Now the following subclasses of $\mathscr{T}_{\text {prog }}$ can be defined. Given $K \in \mathbb{Z}_{+}, B \subseteq \mathbb{R}_{+}$and subsets $R_{0}, \ldots, R_{K}$ of $[0,1)$, let $\mathscr{T}_{\text {prog }}\left(K, B, R_{0}, \ldots, R_{K}\right)$ be the set of all $(K+1)$-bracket marginal-rate progressive tax schedules $\left(\alpha_{0},\left(t_{0}, \ldots, t_{K}\right),\left(\bar{y}_{0}, \ldots, \bar{y}_{K}\right)\right) \in \mathscr{T}_{\text {prog }}$ with intercept $\alpha_{0}$, marginal tax rates $t_{0}, \ldots, t_{K}$ with $t_{k} \in R_{k}$ for each $k \in\{0, \ldots, K\}$, and bracket thresholds $\bar{y}_{1}, \ldots, \bar{y}_{K}$, i.e.,

$\mathscr{T}_{\text {prog }}\left(K, B, R_{0}, \ldots, R_{K}\right):=\left\{\left(\alpha_{0},\left(t_{0}, \ldots, t_{K}\right),\left(\bar{y}_{0}, \ldots, \bar{y}_{K}\right)\right) \in \mathscr{T}_{p r o g}: \alpha_{0} \in B\right.$ and $\left.\left(t_{0}, \ldots, t_{K}\right) \in R_{0} \times \cdots \times R_{K}\right\}$

\footnotetext{
${ }^{2}$ Under the agent monotonicity condition, in both cases the vector components are arranged in increasing order. See Carbonell-Nicolau and Llavador (forthcoming).
} 
Let $\mathfrak{D}$ be the set of all $\left(B,\left(R_{k}\right)_{k=0}^{\infty}\right)$ with $B \subseteq \mathbb{R}$ and $R_{k} \subseteq[0,1)$ for each $k$. For each $\left(B,\left(R_{k}\right)\right) \in \mathfrak{D}$, define

$$
\mathscr{T}_{\text {prog }}\left(B,\left(R_{k}\right)\right):=\bigcup_{K \in \mathbb{Z}_{+}} \mathscr{T}_{\text {prog }}\left(K, B, R_{0}, \ldots, R_{K}\right)
$$

When $R_{0}=R_{1}=\cdots=R$, we write $\mathscr{T}_{\text {prog }}(B, R)$ for $\mathscr{T}_{\text {prog }}\left(B,\left(R_{k}\right)\right)$.

We now recapture a result from Carbonell-Nicolau and Llavador (forthcoming).

Theorem 1 (Carbonell-Nicolau and Llavador (forthcoming, Theorem 1)). Given $u \in \mathscr{U}^{*}, a$ tax schedule in $\mathscr{T}$ is u-iir only if it is marginal-rate progressive.

Definition 5. A tax schedule $T \in \mathscr{T}$ is linear if $T(y)=-b+r y$ for all $y \in \mathbb{R}_{+}$and some $b \geq 0$ and $r \in[0,1)$.

The set of all linear tax schedules in $\mathscr{T}$ is denoted by $\mathscr{T}_{\text {lin }}$. Given $B \subseteq \mathbb{R}_{+}$and $R \subseteq[0,1)$, define

$$
\mathscr{T}_{\text {lin }}(B, R):=\left\{-b+r y \in \mathscr{T}_{\text {lin }}:(b, r) \in B \times R\right\}
$$

Given $u \in \mathscr{U}$, let $\mathscr{T}_{u \text {-iir }}$ be the set of all $u$-iir tax schedules in $\mathscr{T}$.

The following result generalizes Theorem 2 in Carbonell-Nicolau and Llavador (forthcoming). It can be proven using an adaptation of the proof of Theorem 2 in Carbonell-Nicolau and Llavador (forthcoming). The details are provided in the Appendix.

For $B \subseteq \mathbb{R}_{+}$, define

$$
\bar{B}:=\bigcup_{b \in B}\left\{b^{\prime} \in \mathbb{R}_{+}: b^{\prime} \geq b\right\} .
$$

Theorem 2. Given $u \in \mathscr{U}^{*}$ and $\left(B,\left(R_{k}\right)\right) \in \mathfrak{D}, \mathscr{T}_{\text {prog }}\left(\bar{B},\left(R_{k}\right)\right) \subseteq \mathscr{T}_{u \text {-iir }} \subseteq \mathscr{T}_{\text {prog }}$ if and only if the members of $\mathscr{T}_{\text {lin }}\left(\bar{B}, \bigcup_{k} R_{k}\right)$ are $u$-iir.

For $B=\mathbb{R}_{+}$and $R_{k}=[0,1)$ for each $k$, one has $\mathscr{T}_{\text {prog }}\left(B,\left(R_{k}\right)\right)=\mathscr{T}_{\text {prog }}$ and $\mathscr{T}_{\text {lin }}\left(\bar{B}, \bigcup_{k} R_{k}\right)=$ $\mathscr{T}_{\text {lin }}$, and Theorem 2 immediately gives Theorem 2 in Carbonell-Nicolau and Llavador (forthcoming).

Corollary 1 (to Theorem 2). Given $u \in \mathscr{U}^{*}, \mathscr{T}_{u-i i r}=\mathscr{T}_{\text {prog }}$ if and only if the members of $\mathscr{T}_{\text {lin }}$ are $u$-iir.

When $T$ is a linear tax schedule in $\mathscr{T}_{\text {lin }}$ with $T(y)=-b$, where $b \geq 0$, we write $l^{u}(a, b)$ for $l^{u}(a, T)$. For each $(a, b) \in \mathbb{R}_{++} \times \mathbb{R}_{+}, l^{u}(a, b)$ is a solution to the problem

$$
\max _{l \in[0,1]} u(a l+b, l)
$$

Since $u$ is strictly quasiconcave on $\mathbb{R}_{+_{+}} \times[0,1)$, for each $(a, b) \in \mathbb{R}_{+_{+}} \times \mathbb{R}_{+}$, there is a unique solution $l^{u}(a, b)$ to (3). For given $b \geq 0$, the derivative of the map $a \mapsto l^{u}(a, b)$ exists for all but perhaps one $a>0$ (see Carbonell-Nicolau and Llavador (forthcoming)).

For $(a, b) \in \mathbb{R}_{++} \times \mathbb{R}_{+}$, define

$$
\zeta^{u}(a, b):=\frac{\partial\left(a l^{u}(a, b)+b\right)}{\partial a} \cdot \frac{a}{a l^{u}(a, b)+b}
$$


this is the elasticity of income with respect to ability at ability level $a$ and endowment $b$.

Given $B \subseteq \mathbb{R}_{+}$and $R \subseteq[0,1)$, let $\mathscr{U}(B, R)$ be the set of all $u \in \mathscr{U}^{*}$ satisfying the following condition:

$$
\zeta^{u}((1-r) a, b) \leq \zeta^{u}(a, 0), \quad \text { for all }(a, b, r) \in \mathbb{R}_{++} \times B \times R \text {. }
$$

The following result generalizes Theorem 3 in Carbonell-Nicolau and Llavador (forthcoming). The proof is relegated to the Appendix.

Theorem 3. For $u \in \mathscr{U}^{*}$, the members of $\mathscr{T}_{\text {lin }}(B, R)$ are $u$-iir if and only if $u \in \mathscr{U}(B, R)$.

Let $\widehat{\mathscr{U}}$ denote the class of utility functions $u \in \mathscr{U}^{*}$ satisfying the following two conditions:

(i) $\zeta^{u}(a, b) \leq \zeta^{u}(a, 0)$ for all $(a, b) \in \mathbb{R}_{++} \times \mathbb{R}_{+}$; and

(ii) the map $a \mapsto \zeta^{u}(a, 0)$ defined on $\mathbb{R}_{++}$is nondecreasing.

From the proof of Theorem 3 in Carbonell-Nicolau and Llavador (forthcoming), $\mathscr{U}\left(\mathbb{R}_{+},[0,1)\right)=$ $\widehat{\mathscr{U}}$, and in this case Theorem 3 immediately gives Theorem 3 in Carbonell-Nicolau and Llavador (forthcoming).

Corollary 2 (to Theorem 3). For $u \in \mathscr{U}^{*}$, the members of $\mathscr{T}_{\text {lin }}$ are $u$-iir if and only if $u \in \widehat{\mathscr{U}}$.

Now combining Theorem 2 and Theorem 3 yields the following result, which refines Corollary 3 in Carbonell-Nicolau and Llavador (forthcoming).

Theorem 4. Given $u \in \mathscr{U}^{*}$ and $\left(B,\left(R_{k}\right)\right) \in \mathfrak{D}, \mathscr{T}_{\text {prog }}\left(\bar{B},\left(R_{k}\right)\right) \subseteq \mathscr{T}_{u-i i r} \subseteq \mathscr{T}_{\text {prog }}$ if and only if $u \in \mathscr{U}\left(\bar{B}, \cup_{k} R_{k}\right)$.

\section{Applications}

In this section we characterize the subclasses of progressive taxes that are inequality reducing for two commonly used families of income-leisure preferences: the CES and the quasilinear preferences. The CES utility function (often in its Cobb-Douglas version) is very common in the literature on life-cycle models (Heckman and MaCurdy, 1982; French, 2005; Blundell et al., 2016), while static models with fixed costs traditionally work with quasi-linear preferences (Cogan, 1981). ${ }^{3}$ These utilities are also dominant in surveys and textbooks on labor supply and fiscal policy (Pencavel, 1986; Killingsworth and Heckman, 1986; Auerbach and Kotlikoff, 1987; Keane, 2011; Blundell et al., 2016).

\subsection{CES utility}

Consider the well-know CES utility function

$$
u(c, l):= \begin{cases}c^{\gamma}+\beta(1-l)^{\gamma} & \text { if } \gamma \in(0,1) \\ -c^{\gamma}-\beta(1-l)^{\gamma} & \text { if } \gamma<0\end{cases}
$$

\footnotetext{
${ }^{3}$ Static models tend to specify a labor supply function directly, which makes it difficult to identify a widely used utility function (Keane, 2011, page 966).
} 
where $\beta$ is a positive constant. One has

$$
\begin{gathered}
l^{u}(a, b)= \begin{cases}\frac{\left(\frac{a}{\beta}\right)^{\frac{1}{1-\gamma}}-b}{a+\left(\frac{a}{\beta}\right)^{\frac{1}{1-\gamma}}} & \text { if }\left(\frac{a}{\beta}\right)^{\frac{1}{1-\gamma}} \geq b, \\
0 & \text { otherwise, }\end{cases} \\
a l^{u}(a, b)+b= \begin{cases}\frac{\left(\frac{a}{\beta}\right)^{\frac{1}{1-\gamma}}(a+b)}{a+\left(\frac{a}{\beta}\right)^{\frac{1}{1-\gamma}}} & \text { if }\left(\frac{a}{\beta}\right)^{\frac{1}{1-\gamma}} \geq b, \\
b & \text { otherwise },\end{cases} \\
\zeta^{u}(a, 0)= \begin{cases}\frac{(1-\gamma) a^{\frac{2}{1-\gamma}}+a^{\frac{2-\gamma}{1-\gamma}} \beta^{\frac{1}{1-\gamma}}}{(1-\gamma) a^{\frac{2}{1-\gamma}}+(1-\gamma) a^{\frac{2-\gamma}{1-\gamma}} \beta^{\frac{1}{1-\gamma}}} & \text { if }\left(\frac{a}{\beta}\right)^{\frac{1}{1-\gamma}} \geq b, \\
0 & \text { otherwise, }\end{cases}
\end{gathered}
$$

and

$$
\zeta^{u}((1-r) a, b)= \begin{cases}\frac{(1-\gamma)((1-r) a)^{\frac{2}{1-\gamma}}+\beta^{\frac{1}{1-\gamma}} b \gamma((1-r) a)^{\frac{1}{1-\gamma}}+\beta^{\frac{1}{1-\gamma}}((1-r) a)^{\frac{2-\gamma}{1-\gamma}}}{(1-\gamma)((1-r) a)^{\frac{\gamma}{1-\gamma}}((1-r) a+b)\left[(1-r) a \beta^{\frac{1}{1-\gamma}}+((1-r) a)^{\frac{1}{1-\gamma}}\right]} & \text { if }\left(\frac{(1-r) a}{\beta}\right)^{\frac{1}{1-\gamma}} \geq b, \\ 0 & \text { otherwise. }\end{cases}
$$

The following result can be proven for the specification in (4). Intuition for the result is presented after the formal proof.

Proposition 1. Let $u$ be the CES utility function given in (4). Suppose that $R \subseteq[0,1)$ and $\sup R<1$. Then there exists $\underline{b} \geq 0$ such that $\mathscr{T}_{\text {prog }}\left(B^{*}, R\right) \subseteq \mathscr{T}_{u \text {-iir }} \subseteq \mathscr{T}_{\text {prog }}$ if and only if $\gamma \in\left[\frac{1}{2}, 1\right)$, where $B^{*}:=\left\{b \in \mathbb{R}_{+}: b \geq \underline{b}\right\}$.

Proof. Since $u \in \mathscr{U}^{*}$, given $\underline{b} \geq 0, B^{*}:=\left\{b \in \mathbb{R}_{+}: b \geq \underline{b}\right\}$, and $R \subseteq[0,1)$, Theorem 4 gives $\mathscr{T}_{\text {prog }}\left(B^{*}, R\right) \subseteq \mathscr{T}_{u \text {-iir }} \subseteq \mathscr{T}_{\text {prog }}$ if and only if $u \in \mathscr{U}\left(B^{*}, R\right)$.

Given $(a, b, r) \in \mathbb{R}_{++} \times B^{*} \times R$, if $\left(\frac{a}{\beta}\right)^{\frac{1}{1-\gamma}}<b$, then $\left(\frac{(1-r) a}{\beta}\right)^{\frac{1}{1-\gamma}}<b$, implying $\zeta^{u}(a, 0)=0 \geq$ $0=\zeta^{u}((1-r) a, b)$. If $\left(\frac{a}{\beta}\right)^{\frac{1}{1-\gamma}} \geq b>\left(\frac{(1-r) a}{\beta}\right)^{\frac{1}{1-\gamma}}$, then $\zeta^{u}(a, 0) \geq 0=\zeta^{u}((1-r) a, b)$. If $\left(\frac{a}{\beta}\right)^{\frac{1}{1-\gamma}} \geq$ $\left(\frac{(1-r) a}{\beta}\right)^{\frac{1}{1-\gamma}} \geq b$, then $\zeta^{u}(a, 0) \geq \zeta^{u}((1-r) a, b)$ if and only if

$$
\frac{(1-\gamma) a^{\frac{2}{1-\gamma}}+a^{\frac{2-\gamma}{1-\gamma}} \beta^{\frac{1}{1-\gamma}}}{(1-\gamma) a^{\frac{2}{1-\gamma}}+(1-\gamma) a^{\frac{2-\gamma}{1-\gamma}} \beta^{\frac{1}{1-\gamma}}} \geq \frac{(1-\gamma)((1-r) a)^{\frac{2}{1-\gamma}}+\beta^{\frac{1}{1-\gamma}} b \gamma((1-r) a)^{\frac{1}{1-\gamma}}+\beta^{\frac{1}{1-\gamma}}((1-r) a)^{\frac{2-\gamma}{1-\gamma}}}{(1-\gamma)((1-r) a)^{\frac{\gamma}{1-\gamma}}((1-r) a+b)\left[(1-r) a \beta^{\frac{1}{1-\gamma}}+((1-r) a)^{\frac{1}{1-\gamma}}\right]}
$$


Arranging terms gives

$$
\begin{array}{r}
a^{\frac{1}{1-\gamma}}(a(1-r))^{\frac{1}{1-\gamma}}\left[(a(1-r))^{-1}\left((1-\gamma) a^{\frac{1}{1-\gamma}}+a \beta^{\frac{1}{1-\gamma}}\right)\left(a(1-r) \beta^{\frac{1}{1-\gamma}}+(a(1-r))^{\frac{1}{1-\gamma}}\right)\right. \\
\left.-\gamma \beta^{\frac{1}{1-\gamma}}\left(a^{\frac{1}{1-\gamma}}+a \beta^{\frac{1}{1-\gamma}}\right)\right] b \\
\geq a^{\frac{1}{1-\gamma}}(a(1-r))^{\frac{1}{1-\gamma}}\left[\left(a^{\frac{1}{1-\gamma}}+a \beta^{\frac{1}{1-\gamma}}\right)\left(a(1-r) \beta^{\frac{1}{1-\gamma}}+(1-\gamma)(a(1-r))^{\frac{1}{1-\gamma}}\right)\right. \\
\left.-\left((1-\gamma) a^{\frac{1}{1-\gamma}}+a \beta^{\frac{1}{1-\gamma}}\right)\left(a(1-r) \beta^{\frac{1}{1-\gamma}}+(a(1-r))^{\frac{1}{1-\gamma}}\right)\right] .
\end{array}
$$

This simplifies to

$$
\begin{array}{r}
{\left[\left((1-\gamma) a^{\frac{1}{1-\gamma}}+a \beta^{\frac{1}{1-\gamma}}\right)\left(\beta^{\frac{1}{1-\gamma}}+(a(1-r))^{\frac{\gamma}{1-\gamma}}\right)-\gamma \beta^{\frac{1}{1-\gamma}}\left(a^{\frac{1}{1-\gamma}}+a \beta^{\frac{1}{1-\gamma}}\right)\right] b} \\
\geq a^{1+\frac{1}{1-\gamma}} \beta^{\frac{1}{1-\gamma}} \gamma\left(1-r-(1-r)^{\frac{1}{1-\gamma}}\right) .
\end{array}
$$

We claim that if $\sup R<1$ there exists $\underline{b} \geq 0$ such that $\mathscr{T}_{\text {prog }}\left(B^{*}, R\right) \subseteq \mathscr{T}_{u-i i r} \subseteq \mathscr{T}_{\text {prog }}$ if and only if $\gamma \in\left[\frac{1}{2}, 1\right)$. To see this, it suffices to show that (i) for $\gamma<\frac{1}{2}$ and $\underline{b} \geq 0$, there exists $(a, b, r) \in \mathbb{R}_{++} \times B^{*} \times R$ with $\left(\frac{a}{\beta}\right)^{\frac{1}{1-\gamma}} \geq\left(\frac{(1-r) a}{\beta}\right)^{\frac{1}{1-\gamma}} \geq b$ such that (5) does not hold, and (ii) for $\gamma \in\left[\frac{1}{2}, 1\right)$, there exists $\underline{b} \geq 0$ such that for $(a, b, r) \in \mathbb{R}_{++} \times B^{*} \times R$ with $\left(\frac{a}{\beta}\right)^{\frac{1}{1-\gamma}} \geq\left(\frac{(1-r) a}{\beta}\right)^{\frac{1}{1-\gamma}} \geq b$, (5) holds.

Given $\underline{b} \geq 0$ and $\gamma<0$, the bracketed term on the left-hand side of (5) is positive and the right-hand side of (5) divided by the bracketed term on the left-hand side of (5) converges to infinity as $a$ tends to infinity. Consequently, there exists $(a, b, r) \in \mathbb{R}_{++} \times B^{*} \times R$ with $\left(\frac{a}{\beta}\right)^{\frac{1}{1-\gamma}} \geq\left(\frac{(1-r) a}{\beta}\right)^{\frac{1}{1-\gamma}} \geq b$ such that (5) does not hold. If $0<\gamma<\frac{1}{2}$, the right-hand side of (5) is positive, the bracketed term on the left-hand side of (5) is positive for a large enough, and the right-hand side of (5) divided by the bracketed term on the left-hand side of (5) converges to infinity as $a$ tends to infinity. Therefore, there exists $(a, b, r) \in \mathbb{R}_{++} \times B^{*} \times R$ with $\left(\frac{a}{\beta}\right)^{\frac{1}{1-\gamma}} \geq\left(\frac{(1-r) a}{\beta}\right)^{\frac{1}{1-\gamma}} \geq b$ such that (5) does not hold.

If $\gamma=\frac{1}{2}$, the bracketed term on the left-hand side of (5) is positive and the and the righthand side of (5) divided by the bracketed term on the left-hand side of (5) is increasing in $a$ and converges to $r \beta^{2}$ as $a \rightarrow \infty$. Consequently, it suffices to set $\underline{b}=\beta^{2} \sup R \leq \beta^{2}{ }^{4}$

If $\gamma \in\left(\frac{1}{2}, 1\right)$, the bracketed term on the left-hand side of (5) is positive for large enough $a$, and the right-hand side of (5) divided by the bracketed term on the left-hand side of (5) tends to 0 as $a \rightarrow \infty$, and so if $\sup R<1$ there exists $\underline{b}$ such that for $(a, b, r) \in \mathbb{R}_{++} \times B^{*} \times R$ with $\left(\frac{(1-r) a}{\beta}\right)^{\frac{1}{1-\gamma}} \geq b,(5)$ holds. ${ }^{5}$

The parameter $\gamma$ in the CES utility function (4) determines the elasticity of substitution between consumption and leisure. As $\gamma$ tends to 1, the CES utility function becomes linear

\footnotetext{
${ }^{4}$ For example, if $\beta=1$ and the maximum marginal tax rate is $\frac{1}{2}$, then it suffices to consider the set of all the marginal-rate progressive tax schedules that provide a subsidy of at least $\$ \frac{1}{2}$ for those individuals with no income.

${ }^{5}$ For instance, if $\gamma=\frac{3}{4}, \sup R=0.6$, and $\beta=1$, (5) becomes
}

$$
\left[\left(0.25 a^{4}+a\right)\left(1+(a(1-r))^{3}\right)-0.75\left(a^{4}+a\right)\right] b \geq 0.75 a^{4}(1-r)\left(1-(1-r)^{3}\right) .
$$


and consumption and leisure become perfect substitutes. As $\gamma$ tends to $-\infty$, the indifference curves become "right angles," i.e., the social utility function regards the two goods as perfect complements.

Proposition 1 states that when the elasticity of substitution is large enough, i.e., when consumption and leisure substitute "sufficiently well" for each other, there are (nonempty) subclasses of progressive tax schedules whose members are inequality reducing. Specifically, in this case it suffices to choose a sufficiently large subsidy for a progressive tax schedule to be inequality reducing.

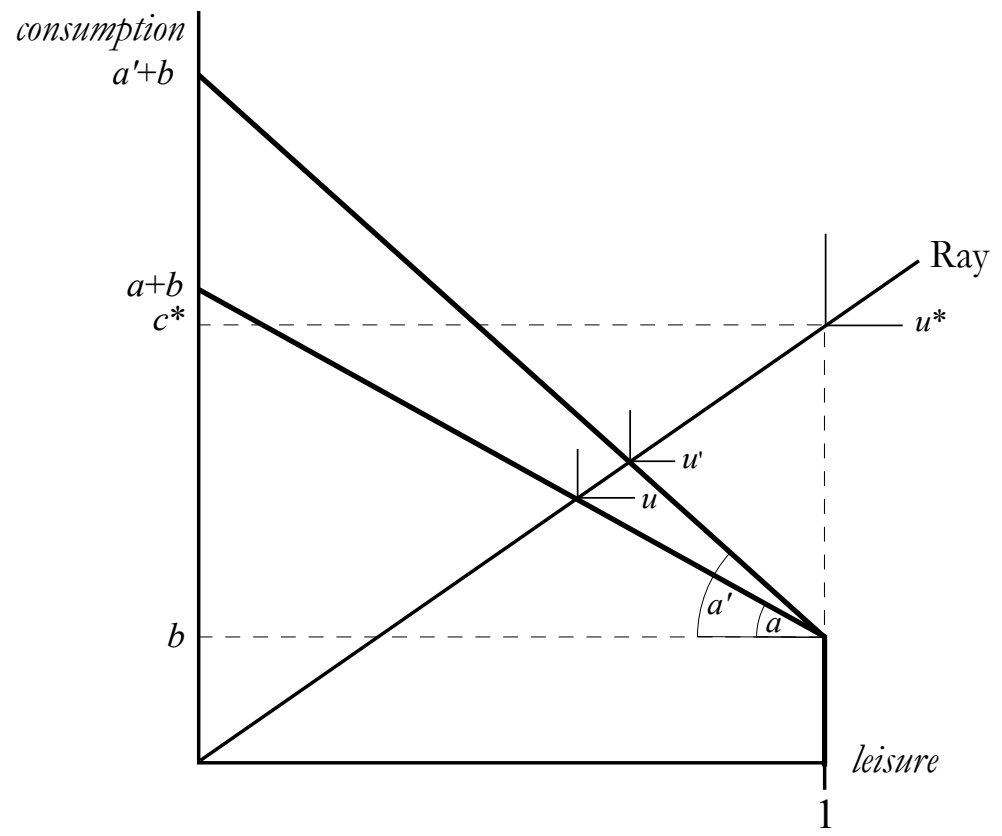

Figure 1: Perfect complements. Individual choice for different ability levels and exogenous income $b$. The ray represents the bundles with the "correct" proportions between leisure and consumption. The maximum utility level $u^{*}$ is attained for the bundle $\left(1, c^{*}\right)$. For sufficiently high abilities, income (the chosen level of consumption) cannot increase at increasing rates. Hence, the elasticity of income to ability must decrease and not all progressive taxes are income inequality reducing (Theorem 3 and Corollary 1 ).

In order to capture the intuition for this result, recall that a necessary and sufficient condition for the containments $\mathscr{T}_{\text {prog }}\left(B^{*}, R\right) \subseteq \mathscr{T}_{u-i i r} \subseteq \mathscr{T}_{\text {prog }}$ to hold is that $u \in \mathscr{U}\left(B^{*}, R\right)$, and consider first the opposite extreme case when consumption and leisure are perfect complements. Because leisure is bounded above by 1 , utility has an upper bound, with $\left(1, c^{*}\right)$ as the optimal bundle, where $c^{*}$ is the "ideal" consumption level corresponding to

If $\underline{b}=81$, then given $(a, b, r) \in \mathbb{R}_{++} \times B^{*} \times R$ with $\left(\frac{(1-r) a}{\beta}\right)^{\frac{1}{1-\gamma}}=(1-r)^{4} a^{4} \geq b \geq \underline{b}$, i.e., $a \geq \frac{b^{\frac{1}{4}}}{1-r} \geq \frac{b^{\frac{1}{4}}}{1-r}=\frac{3}{1-r}$, (6) is equivalent to

$$
b \geq \frac{0.75 a^{4}(1-r)\left(1-(1-r)^{3}\right)}{\left(0.25 a^{4}+a\right)\left(1+(a(1-r))^{3}\right)-0.75\left(a^{4}+a\right)},
$$

and since

$$
\frac{0.75 a^{4}(1-r)\left(1-(1-r)^{3}\right)}{\left(0.25 a^{4}+a\right)\left(1+(a(1-r))^{3}\right)-0.75\left(a^{4}+a\right)}<81
$$

and $b \geq \underline{b}$, it follows that (7) holds. 


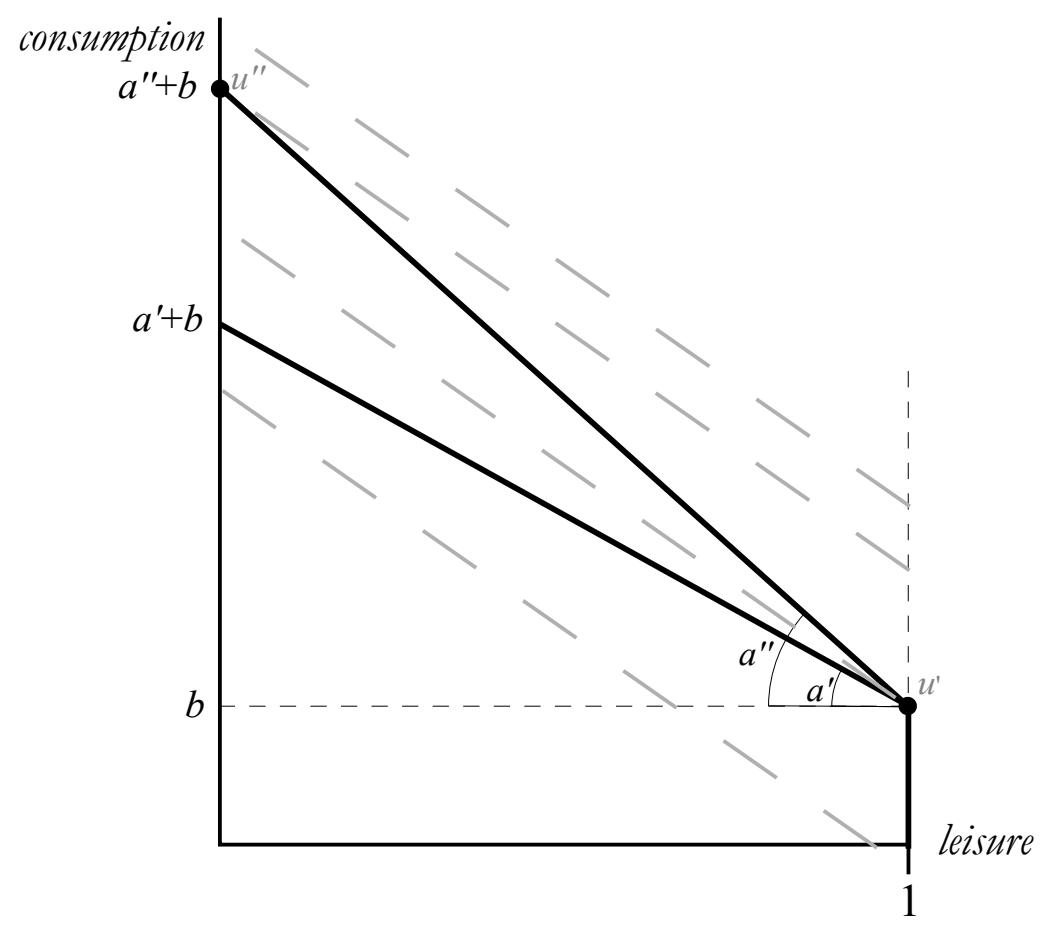

Figure 2: Perfect substitutes. Individual choice for different ability levels and exogenous income $b$. Individuals with a sufficiently high ability, like $a^{\prime \prime}$, choose zero leisure; while those with sufficiently low ability, like $a^{\prime}$, choose zero labor. Hence, the elasticity of income to ability is non-decreasing and all progressive taxes are income inequality reducing (Theorem 3 and Corollary 1).

maximum leisure (see Figure 1). Individuals of higher ability, whose consumption entails a lower opportunity cost in terms of leisure time, choose higher consumption levels. As $a$ grows large, the optimal consumption level converges to $c^{*}$. Hence, for a sufficiently large ability, the change in consumption (and hence in income) with respect to ability increases less than proportionally in ability. This implies that the elasticity of income with respect to ability must decrease at some point, and so $u \notin \mathscr{U}\left(B^{*}, R\right)$.

At the other extreme, when consumption and leisure are treated as perfect substitutes, higher ability individuals will choose zero leisure and low ability individuals zero labor, implying that the elasticity of income with respect to ability is not decreasing (Figure 2).

For non-extreme cases, when consumption and leisure are not good substitutes, one can always find individuals of sufficiently high ability for whom the elasticity of income with respect to ability decreases, as in the case of perfect complements. Alternatively, when consumption and leisure substitute relatively well, the elasticity condition is satisfied for sufficiently high ability levels and can also be guaranteed for low ability agents by equalizing their incomes with a sufficiently large subsidy. Hence the condition $B^{*}$ in the statement of Proposition 1.

Remark 1. When $\gamma \rightarrow 0$ the CES utility function converges to the Cobb-Douglas utility function, and in this limiting case (5) holds for all $(a, b, r) \in \mathbb{R}_{++} \times[0,+\infty) \times[0,1)$, and so 
Theorem 4 gives $\mathscr{T}_{u \text {-iir }}=\mathscr{T}_{\text {prog }}$ (this was established in Carbonell-Nicolau and Llavador (forthcoming, Remark 3)).

\subsection{Quasi-linear utility}

Consider the quasi-linear utility function

$$
u(c, l):=c+\frac{\beta(1-l)^{1-\delta}}{1-\delta}
$$

where $\beta>0$ and $\delta>0$, with $\delta \neq 1 .{ }^{6}$ One has

$$
l^{u}(a, b)= \begin{cases}1-\left(\frac{a}{\beta}\right)^{-1 / \delta} & \text { if } a \geq \beta \\ 0 & \text { if } a \leq \beta\end{cases}
$$

and

$$
a l^{u}(a, b)+b= \begin{cases}a+b-a\left(\frac{a}{\beta}\right)^{-1 / \delta} & \text { if } a \geq \beta \\ b & \text { if } a \leq \beta\end{cases}
$$

Define $\theta(a):=\left(\frac{a}{\beta}\right)^{-1 / \delta}$ and $\theta_{r}(a):=\left(\frac{(1-r) a}{\beta}\right)^{-1 / \delta}$. Note that $\theta(a)>1$ for $a \geq \beta$, and $\theta_{r}(a)>1$ for $a \geq \frac{\beta}{1-r}>\beta$. Compute

$$
\zeta^{u}(a, 0)= \begin{cases}1-\frac{1}{\delta(1-\theta(a))} & \text { if } a \geq \beta \\ 0 & \text { otherwise }\end{cases}
$$

and

$$
\zeta^{u}((1-r) a, b)= \begin{cases}\frac{a(1-r)-\left(1-\theta_{r}(a)\right)(1-r) a \delta}{b \theta_{r}(a) \delta-\left(1-\theta_{r}(a)\right)(1-r) a \delta} & \text { if } a \geq \frac{\beta}{(1-r)} \\ 0 & \text { otherwise }\end{cases}
$$

The following result can be proven for the specification in (8). Intuition is furnished after the formal proof.

Proposition 2. Let $u$ be the quasi-linear utility function given in (8). Suppose that $R \subseteq[0,1$ ) and $\sup R<1$. Then there exists $\underline{b} \geq 0$ such that $\mathscr{T}_{\text {prog }}\left(B^{*}, R\right) \subseteq \mathscr{T}_{u-i i r} \subseteq \mathscr{T}_{\text {prog }}$ if and only if $\delta \in(0,1)$, where $B^{*}:=\left\{b \in \mathbb{R}_{+}: b \geq \underline{b}\right\}$.

Proof. Since $u \in \mathscr{U}^{*}$, given $\underline{b} \geq 0, B^{*}:=\left\{b \in \mathbb{R}_{+}: b \geq \underline{b}\right\}$, and $R \subseteq[0,1)$, Theorem 4 gives $\mathscr{T}_{\text {prog }}\left(B^{*}, R\right) \subseteq \mathscr{T}_{u-i i r} \subseteq \mathscr{T}_{\text {prog }}$ if and only if $u \in \mathscr{U}\left(B^{*}, R\right)$.

Given $(a, b, r) \in \mathbb{R}_{++} \times B^{*} \times R$, if $a \leq \beta /(1-r)$, then $\zeta^{u}((1-r) a, b)=0 \leq \zeta^{u}(a, 0)$. If $a>\beta /(1-r)$, then $\zeta^{u}(a, 0) \geq \zeta^{u}((1-r) a, b)$ if and only if

$$
1-\frac{1}{\delta(1-\theta(a))} \geq \frac{a(1-r)-\left(1-\theta_{r}(a)\right)(1-r) a \delta}{b \theta_{r}(a) \delta-\left(1-\theta_{r}(a)\right)(1-r) a \delta} .
$$

\footnotetext{
${ }^{6}$ The $M R S(c, l)$ tends to $+\infty$ as $l \rightarrow 1^{-}$(recall the Inada condition in (1)) if and only if $\delta>0$.
} 
Using $\theta(a)>\theta_{r}(a)>1$, and arranging terms, we obtain

$$
b\left(\delta(\theta(a)-1) \theta_{r}(a) \delta+\theta_{r}(a) \delta\right) \geq(\theta(a)-1)(1-r) a \delta-\left(\theta_{r}(a)-1\right)(1-r) a \delta .
$$

This simplifies to

$$
b \geq \frac{\left(\theta(a)-\theta_{r}(a)\right)(1-r) a}{(1+\delta(\theta(a)-1)) \theta_{r}(a)}
$$

Finally, since $\theta_{r}(a)=(1-r)^{1 / \delta} \theta(a)=(1-r)^{1 / \delta}\left(\frac{a}{\beta}\right)^{-1 / \delta}$,

$$
b \geq\left((1-r)^{-1 / \delta}-1\right)(1-r) \frac{a}{1+\left(\left(\frac{a}{\beta}\right)^{1 / \delta}-1\right) \delta}=K(r) \varphi(a)
$$

where $K(r):=\left((1-r)^{-1 / \delta}-1\right)(1-r)>0$ for all $r \in(0,1)$, and $\varphi(a):=\frac{a}{1+\left((a / \beta)^{1 / \delta}-1\right) \delta}$ for $a \geq \beta$.

We claim that if $\sup R<1$ there exists $\underline{b} \geq 0$ such that $\mathscr{T}_{\text {prog }}\left(B^{*}, R\right) \subseteq \mathscr{T}_{u-i i r} \subseteq \mathscr{T}_{\text {prog }}$ if and only if $\delta \in(0,1)$. To see this, it suffices to show that (i) for $\delta>1$ and $\underline{b} \geq 0$, there exists $(a, b, r) \in \mathbb{R}_{++} \times B^{*} \times R$ with $a>\beta$ such that (9) does not hold; and (ii) for $\delta \in(0,1)$, there exists $\underline{b} \geq 0$ such that for $(a, b, r) \in[\beta, \infty) \times B^{*} \times R,(9)$ holds.

Given $\underline{b} \geq 0$ and $\delta>1, \varphi(a)$ converges to infinity as $a$ tends to infinity, and consequently, there exists $(a, b, r) \in \mathbb{R}_{++} \times B^{*} \times R$ with $a>\beta$ such that (9) does not hold.

If $\delta \in(0,1), \varphi(a)$ tends to 0 as $a \rightarrow \infty$ and $K(r)$ is monotone increasing. Hence, if $\sup R<1$, there exists $\underline{b}$ such that for $(a, b, r) \in[\beta, \infty) \times B^{*} \times R,(9)$ holds.

For the quasi-linear utility function, the parameter $\delta$ determines the inverse of the elasticity of leisure with respect to ability, so that leisure is elastic for $\delta<1$ and inelastic for $\delta>1 .{ }^{7}$ Proposition 2 states that when the demand of leisure is inelastic, any progressive tax schedule is inequality reducing once an appropriate subsidy is chosen.

The intuition for this result is related to that for the CES utility function. Observe that consumption and leisure become perfect substitutes for $\delta=0$, and that the degree of substitutability decreases as $\delta$ increases. ${ }^{8}$ As $\delta \rightarrow 0$, the demand of leisure becomes perfectly elastic, with consumption and leisure becoming perfect substitutes. High ability individuals choose zero leisure and low ability individuals zero labor, implying that the elasticity of income with respect to ability is not decreasing. (Recall Figure 2.)

For $\delta<1$, leisure demand is elastic and an ability increase leads to a more than proportional leisure decrease, producing a larger increase in income for sufficiently large ability levels, and satisfying the elasticity condition for sufficiently large ability levels. The elasticity condition can also be guaranteed for low ability individuals by equalizing their incomes with a sufficiently large subsidy. Hence the condition $B^{*}$ in the statement of Proposition 1.

\footnotetext{
${ }^{7}$ It is easy to obtain the demand of leisure as $(\alpha / \beta)^{-1 / \delta}$ for $a \geq \beta$, and hence the elasticity of leisure to ability as $-1 / \delta$.

${ }^{8}$ Both the quasilinear utility function considered here and the CES utility function from Subsection 4.1 become the linear utility function $u(c, l)=c+\beta(1-l)$ for $\delta=0$ and $\gamma=1$, respectively.
} 


\section{Concluding remarks}

This paper characterizes social preferences for which various subclasses of progressive tax schedules are inequality reducing. The framework considered here, which subsumes that in Carbonell-Nicolau and Llavador (forthcoming), allows one to expand the set of social preferences by suitably restricting the set of progressive taxes. This is illustrated in Section 4 for two standard families of utility functions: the CES and the quasi-linear utility functions. Indeed, the characterizations in Proposition 1 and Proposition 2 state that when the elasticity of substitution between consumption and leisure is large enough, it suffices to choose a sufficiently large subsidy for a progressive tax schedule to be inequality reducing. In this regard, while the relevant parameter regions may differ across families of social preferences, our discussions in Section 4 suggest that the elasticity of substitution between consumption and leisure crucially determines the existence of nonempty subclasses of inequality reducing tax systems.

\section{Appendix}

In this appendix we present the proofs of Theorem 2 and Theorem 3. Each proof is preceded by a restatement of its corresponding theorem for the convenience of the reader.

The following two lemmas, whose proofs can be found in Carbonell-Nicolau and Llavador (forthcoming) (see Lemma 2 and Lemma 3 in Carbonell-Nicolau and Llavador (forthcoming)), are instrumental in the proofs of Theorem 2 and Theorem 3.

Lemma 1. Given $u \in \mathscr{U},(c, y) \in \mathbb{R}_{++}^{2}$, and $q \in(0,+\infty)$, there exists an a $>y$ such that $\eta^{a}(c, y)=$ $q$.

Lemma 2. Given $u \in \mathscr{U}^{*}$, a tax schedule $T \in \mathscr{T}$ is $u$-iir if and only if for any ability distribution $\boldsymbol{a} \in \mathscr{A}$ and for any pre-tax and post-tax income functions $y^{u}$ and $x^{u}$,

$$
\frac{x^{u}\left(a_{i}, T\right)}{y^{u}\left(a_{i}, 0\right)} \geq \frac{x^{u}\left(a_{i+1}, T\right)}{y^{u}\left(a_{i+1}, 0\right)} \quad \forall i \in\{1, \ldots, n-1\}: y^{u}\left(a_{i}, 0\right)>0 .
$$

\section{A Proof of Theorem 2}

Theorem 2. Given $u \in \mathscr{U}^{*}$ and $\left(B,\left(R_{k}\right)\right) \in \mathfrak{D}, \mathscr{T}_{\text {prog }}\left(\bar{B},\left(R_{k}\right)\right) \subseteq \mathscr{T}_{u-i i r} \subseteq \mathscr{T}_{\text {prog }}$ if and only if the members of $\mathscr{T}_{\text {lin }}\left(\bar{B}, \bigcup_{k} R_{k}\right)$ are u-iir.

Proof. ${ }^{9}$ Suppose that $u \in \mathscr{U}^{*}$ and $\left(B,\left(R_{k}\right)\right) \in \mathfrak{D}$.

Since $\mathscr{T}_{\text {lin }}\left(\bar{B}, \bigcup_{k} R_{k}\right) \subseteq \mathscr{T}_{\text {prog }}\left(\bar{B},\left(R_{k}\right)\right)$, the 'only if' part of the equivalence is obvious.

Assume now that the members of $\mathscr{T}_{\text {lin }}\left(\bar{B}, \bigcup_{k} R_{k}\right) \subseteq \mathscr{T}_{\text {u-iir }}$. We need to prove that $\mathscr{T}_{\text {prog }}\left(\bar{B},\left(R_{k}\right)\right) \subseteq$

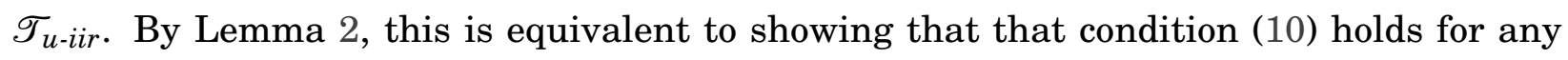
$T \in \mathscr{T}_{\text {prog }}\left(\bar{B},\left(R_{k}\right)\right)$, for any ability distribution $\boldsymbol{a} \in \mathscr{A}$, and for any pre-tax and post-tax income functions $y^{u}$ and $x^{u}$.

\footnotetext{
${ }^{9}$ This proof is an adaptation of the proof for Theorem 2 in Carbonell-Nicolau and Llavador (forthcoming).
} 


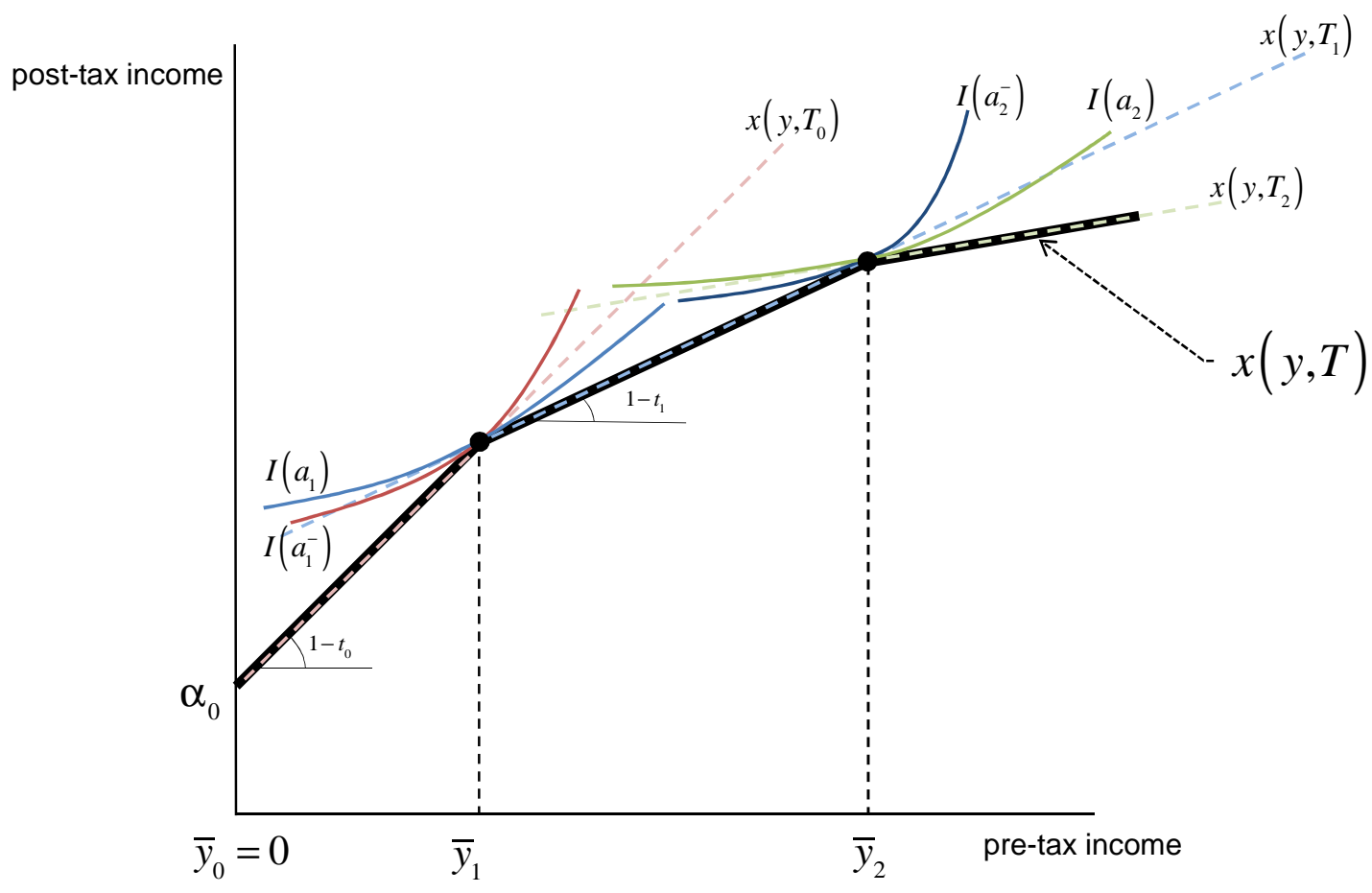

Figure 3: Figure for Theorem 2

Take $T=\left(\alpha_{0}, \boldsymbol{t}, \overline{\boldsymbol{y}}\right) \in \mathscr{T}_{\text {prog }}\left(\bar{B},\left(R_{k}\right)\right)$ and, for each income threshold $\bar{y}_{k}$ of $T$, define the linear tax schedule $T_{k}(y):=t_{k} y-\alpha_{k}$ with $\alpha_{0} \in \bar{B}, t_{k} \in R_{k}$ for $k \in\{0, \ldots, K\}$, and $\alpha_{k}:=\alpha_{k-1}+\left(t_{k}-t_{k-1}\right) \bar{y}_{k}$ for $k \in\{1, \ldots, K\}$.

Pre-tax and post-tax income functions, $y^{u}$ and $x^{u}$, are uniquely defined, since preferences are strictly quasiconcave and the tax function $T$ is convex. For $k \in\{1, \ldots, K\}$, define the abilities $a_{k}^{-}$and $a_{k}$ such that

$$
a_{k}^{-}:=\min \left\{a: y^{u}\left(a, T_{k-1}\right)=\bar{y}_{k}^{u}\right\} \quad \text { and } \quad a_{k}:=\max \left\{a: y\left(a, T_{k}\right)=\bar{y}_{k}\right\}
$$

(see Figure 3). Lemma 1 guarantees that $a_{k}^{-}$and $a_{k}$ exist and are well-defined for all $k \in\{1, \ldots, K\}$.

Furthermore, since $T$ is marginal-rate progressive (and hence $t_{k-1}<t_{k}$ for all $k \in$ $\{1, \ldots, K\}$ ), agent monotonicity (Definition 2) implies that $a_{k}^{-} \leq a_{k}<a_{k+1}^{-}$.

Next, define the following family of sets covering $(0,+\infty)$ :

$$
\mathfrak{A}:=\left\{\left(0, a_{1}^{-}\right],\left\{\left[a_{k}^{-}, a_{k}\right]\right\}_{k=1}^{K},\left\{\left[a_{k}, a_{k+1}^{-}\right]\right\}_{k=1}^{K-1},\left[a_{K},+\infty\right)\right\} .
$$

We first show that condition (10) is satisfied for ability distributions contained in each element of the family $\mathfrak{A}$. 
(i) Consider first the interval $\left(0, a_{1}^{-}\right]$. Observe that $y^{u}(a, T)=y^{u}\left(a, T_{0}\right)$ for all $a \leq a_{1}^{-}$. Because $T_{0}$ is a linear tax, it is $u$-iir, and so Lemma 2 gives

$$
\frac{x^{u}(a, T)}{y^{u}(a, 0)}=\frac{x^{u}\left(a, T_{0}\right)}{y^{u}(a, 0)} \geq \frac{x^{u}\left(a^{\prime}, T_{0}\right)}{y^{u}\left(a^{\prime}, 0\right)}=\frac{x^{u}\left(a^{\prime}, T\right)}{y^{u}\left(a^{\prime}, 0\right)} \quad \forall a \leq a^{\prime} \leq a_{1}^{-} .
$$

(ii) For $\left[a_{K},+\infty\right)$, a symmetric argument shows that

$$
\frac{x^{u}(a, T)}{y^{u}(a, 0)} \geq \frac{x^{u}\left(a^{\prime}, T\right)}{y^{u}\left(a^{\prime}, 0\right)} \quad \forall a_{K} \leq a \leq a^{\prime}
$$

(iii) Now consider the interval $\left[a_{k}^{-}, a_{k}\right]$ for $k \in\{1, \ldots, K\}$. Observe that

$$
y^{u}\left(a_{k}, T\right)=y^{u}\left(a_{k}, T_{k}\right)=\bar{y}_{k}=y^{u}\left(a_{k}^{-}, T_{k-1}\right)=y^{u}\left(a_{k}^{-}, T\right) .
$$

Because the map $a \mapsto y^{u}(a, T)$ is monotone (Mirrlees, 1971, Theorem 1), $y^{u}(a, T)=\bar{y}_{k}$ for all $a \in\left[a_{k}^{-}, a_{k}\right]$, and $y^{u}\left(a^{\prime}, 0\right) \geq y^{u}(a, 0)$ for all $a_{k}^{-} \leq a \leq a^{\prime} \leq a_{k}$. Therefore,

$$
\frac{x^{u}(a, T)}{y^{u}(a, 0)}=\frac{\bar{y}_{k}-T\left(\bar{y}_{k}\right)}{y^{u}(a, 0)} \geq \frac{\bar{y}_{k}-T\left(\bar{y}_{k}\right)}{y^{u}\left(a^{\prime}, 0\right)}=\frac{x^{u}\left(a^{\prime}, T\right)}{y^{u}\left(a^{\prime}, 0\right)} \quad \forall a, a^{\prime} \in\left[a_{k}^{-}, a_{k}\right], a \leq a^{\prime} .
$$

(iv) Finally, consider the interval $\left[a_{k}, a_{k+1}^{-}\right]$for $k \in\{1, \ldots, K-1\}$. By construction, we have $y^{u}(a, T)=y^{u}\left(a, T_{k}\right)$ for all $a \in\left[a_{k}, a_{k+1}^{-}\right]$. Therefore, since $T_{k}$ is a linear tax in $\mathscr{T}_{\text {lin }}\left(\left(\bar{B}, \cup_{k} R_{k}\right)\right.$, and hence $u$-iir, Lemma 2 gives

$$
\frac{x^{u}(a, T)}{y^{u}(a, 0)}=\frac{x^{u}\left(a, T_{k}\right)}{y^{u}(a, 0)} \geq \frac{x^{u}\left(a^{\prime}, T_{k}\right)}{y^{u}\left(a^{\prime}, 0\right)}=\frac{x^{u}\left(a^{\prime}, T\right)}{y^{u}\left(a^{\prime}, 0\right)} \quad \forall a, a^{\prime} \in\left[a_{k}, a_{k+1}^{-}\right], a \leq a^{\prime} .
$$

Combining equations (11)-(14) we obtain (10) for every $\boldsymbol{a} \in \mathscr{A}$.

\section{B Proof of Theorem 3}

Theorem 3. For $u \in \mathscr{U}^{*}$, the members of $\mathscr{T}_{\text {lin }}(B, R)$ are $u$-iir if and only if $u \in \mathscr{U}(B, R)$.

Proof. Given $u \in \mathscr{U}^{*}, B \subseteq \mathbb{R}_{+}$, and $R \subseteq[0,1)$, the members $T(y)=-b+r y$ of $\mathscr{T}_{\text {lin }}(B, R)$ are $u$-iir if and only if the map

$$
a \mapsto \frac{x^{u}(a, T)}{y^{u}(a, 0)}=\frac{a(1-r) l^{u}(a, T)+b}{a l^{u}(a, 0)}=\frac{a(1-r) l^{u}((1-r) a, b)+b}{a l^{u}(a, 0)}
$$


defined on $\mathbb{R}_{++}$is nonincreasing for every $(b, r) \in B \times R$ (Lemma 2). Equivalently, the members of $\mathscr{T}_{\text {lin }}(B, R)$ are $u$-iir if and only if

$$
\begin{gathered}
\frac{(1-r)\left((1-r) a^{\prime} \frac{\partial l^{u}\left((1-r) a^{\prime}, b\right)}{\partial a}+l^{u}\left((1-r) a^{\prime}, b\right)\right) a^{\prime} l^{u}\left(a^{\prime}, 0\right)}{\left(a^{\prime} l^{u}\left(a^{\prime}, 0\right)\right)^{2}} \\
-\frac{\left((1-r) a^{\prime} l^{u}\left((1-r) a^{\prime}, b\right)+b\right)\left(a^{\prime} \frac{\partial l^{u}\left(a^{\prime}, 0\right)}{\partial a}+l^{u}\left(a^{\prime}, 0\right)\right)}{\left(a^{\prime} l^{u}\left(a^{\prime}, 0\right)\right)^{2}} \leq 0
\end{gathered}
$$

for every $\left(a^{\prime}, b, r\right) \in \mathbb{R}_{++} \times B \times R .{ }^{10}$ Since the above inequality can be expressed as

$$
\frac{(1-r) a^{\prime}\left((1-r) a^{\prime} \frac{\partial l^{u}\left((1-r) a^{\prime}, b\right)}{\partial a}+l^{u}\left((1-r) a^{\prime}, b\right)\right)}{(1-r) a^{\prime} l^{u}\left((1-r) a^{\prime}, b\right)+b} \leq \frac{a^{\prime}\left(a^{\prime} \frac{\partial l^{u}\left(a^{\prime}, 0\right)}{\partial a}+l^{u}\left(a^{\prime}, 0\right)\right)}{a^{\prime} l^{u}\left(a^{\prime}, 0\right)}
$$

or, equivalently, as

$$
\zeta^{u}\left((1-r) a^{\prime}, b\right) \leq \zeta^{u}\left(a^{\prime}, 0\right)
$$

we see that the members of $\mathscr{T}_{\text {lin }}(B, R)$ are $u$-iir if and only if (B) holds for every $\left(a^{\prime}, b, r\right) \in$ $\mathbb{R}_{++} \times B \times R$. Consequently, for $u \in \mathscr{U}^{*}$, the members of $\mathscr{T}_{\text {lin }}(B, R)$ are $u$-iir if and only if $u \in \mathscr{U}(B, R)$.

\section{References}

Allingham, M. (1979) "Inequality and progressive taxation: An example," Journal of Public Economics, Vol. 11, pp. 273-274, DOI: http://dx.doi.org/10.1016/0047-2727 (79) 90009-4.

Auerbach, Alan J. and Laurence J. Kotlikoff (1987) Dynamic fiscal policy, New York: Cambridge University Press.

Blundell, Richard, Eric French, and G. Tetlow (2016) "Retirement Incentives and Labor Supply," in John Piggot and Alan Woodland eds. Handbook of the Economics of Population Aging, Vol. 1A: Elsevier, 1st edition, Chap. 8, pp. 458-556.

Carbonell-Nicolau, O. and H. Llavador (forthcoming) "Inequality reducing properties of progressive income tax schedules: The case of endogenous income," Theoretical Economics.

Cogan, John F. (1981) "Fixed Costs and Labor Supply," Econometrica, Vol. 49, p. 945, DOI: http://dx.doi.org/10.2307/1912512.

Ebert, U. and P. Moyes (2000) "Consistent income tax structures when households are heterogeneous," Journal of Economic Theory, Vol. 90, pp. 116-150, DOI: http://dx. doi. org/10.1006/jeth.1999.2582.

\footnotetext{
${ }^{10}$ More precisely, the map defined in (15) is nonincreasing for every $(b, r) \in B \times R$ if and only if for every $(b, r) \in B \times R,(16)$ holds for all but perhaps one $a^{\prime}>0$.
} 
(2003) "The difficulty of income redistribution with labour supply," Economics Bulletin, Vol. 8, pp. 1-9.

(2007) "Income taxation with labor responses," Journal of Public Economic Theory, Vol. 9, pp. 653-682, DOI: http://dx.doi.org/10.1111/j.1467-9779.2007.00324.x.

Eichhorn, W., H. Funke, and W.F. Richter (1984) "Tax progression and inequality of income distribution," Journal of Mathematical Economics, Vol. 13, pp. 127-131, DOI: http://dx . doi .org/10.1016/0304-4068(84)90012-0.

Fellman, J. (1976) "The effect of transformations of Lorenz curves," Econometrica, Vol. 44, pp. 823-824, DOI: http://dx.doi.org/10.2307/1913450.

Formby, J.P., W. James Smith, and D. Sykes. (1986) "Income redistribution and local tax progressivity: A reconsideration," Canadian Journal of Economics, Vol. 19, pp. 808-811, DOI: http://dx.doi.org/10.2307/135328.

French, Eric (2005) "The Effects of Health, Wealth, and Wages on Labour Supply and Retirement Behavior," Review of Economic Studies, Vol. 72, pp. 395-427.

Heckman, James J. and Thomas MaCurdy (1982) "Corrigendum on A Life Cycle Model of Female Labour Supply," The Review of Economic Studies, Vol. 49, p. 659, DOI: http: //dx.doi.org/10.2307/2297295.

Hemming, R. and M.J. Keen (1983) "Single-crossing conditions in comparisons of tax progressivity," Journal of Public Economics, Vol. 20, pp. 373-380, DOI: http: //dx . doi .org/ $10.1016 / 0047-2727$ (83) 90032-4.

Jakobsson, U. (1976) "On the measurement of the degree of progression," Journal of Public Economics, Vol. 5, pp. 161-168, DOI: http://dx.doi.org/10.1016/0047-2727(76) 90066-9.

Ju, B.-G. and J.D. Moreno-Ternero (2008) "On the equivalence between progressive taxation and inequality reduction," Social Choice and Welfare, Vol. 30, pp. 561-569, DOI: http: //dx.doi.org/10.1007/s00355-007-0254-z.

Kakwani, N.C. (1977) "Applications of Lorenz curves in economic analysis," Econometrica, Vol. 45, pp. 719-728, DOI: http://dx.doi.org/10.2307/1911684.

Keane, Michael P (2011) “Labor Supply and Taxes: A Survey," Journal of Economic Literature, Vol. 49, pp. 961-1075, DOI: http://dx.doi.org/10.1257/jel.49.4.961.

Killingsworth, Mark R. and James J. Heckman (1986) "Female labor supply: A survey," in Orley C. Ashenfelter and Richard Layard eds. Handbook of Labor Economics, Vol. 1, Chap. 2, pp. 103-204, DOI: http://dx.doi.org/10.1016/S1573-4463(86)01005-2. 
Latham, R. (1988) "Lorenz-dominating income tax functions," International Economic Review, Vol. 29, pp. 185-198, DOI: http://dx.doi.org/10.2307/2526818.

Le Breton, M., P. Moyes, and A. Trannoy (1996) "Inequality reducing properties of composite taxation," Journal of Economic Theory, Vol. 69, pp. 71-103, DOI: http://dx .doi .org/10. $1006 /$ jeth. 1996.0038.

Liu, P.-W. (1985) "Lorenz domination and global tax progressivity," Canadian Journal of Economics, Vol. 18, pp. 395-399, DOI: http://dx.doi.org/10.2307/135143.

Mirrlees, J.A. (1971) "Exploration in the theory of optimum income taxation," Review of Economic Studies, Vol. 38, pp. 175-208, DOI: http://dx.doi .org/10.2307/2296779.

Moyes, P. (1988) "A note on minimally progressive taxation and absolute income inequality," Social Choice and Welfare, Vol. 5, pp. 227-234, DOI: http://dx.doi.org/10.1007/ BF00735763.

(1994) "Inequality reducing and inequality preserving transformations of incomes: Symmetric and individualistic transformations," Journal of Economic Theory, Vol. 63, pp. 271-298, DOI: http://dx.doi.org/10.1006/jeth.1994.1043.

Musgrave, R.A. and T. Thin (1948) "Income tax progression, 1929-48," Journal of Political Economy, Vol. 56, pp. 498-514, DOI: http://dx.doi.org/10.1086/256742.

Pencavel, John (1986) "Labor supply of men: A survey," in Handbook of Labor Economics, Vol. 1, Chap. 1, pp. 3-102, DOI: http://dx.doi.org/10.1016/S1573-4463 (86) 01004-0.

Seade, J. (1982) "On the sign of the optimum marginal income tax," Review of Economic Studies, Vol. 49, pp. 637-643, DOI: http://dx.doi.org/10.2307/2297292.

Thistle, P.D. (1988) "Uniform progressivity, residual progression, and single-crossing," Journal of Public Economics, Vol. 37, pp. 121-126, DOI: http://dx.doi.org/10.1016/ 0047-2727 (88) 90009-6.

Thon, D. (1987) "Redistributive properties of progressive taxation," Mathematical Social Sciences, Vol. 14, pp. 185-191, DOI: http://dx.doi.org/10.1016/0165-4896 (87) 90021-7. 